\title{
Disponibilidade de nutrientes no solo, qualidade de grãos e produtividade da soja em solo adubado com lodo de esgoto
}

\author{
Rosana Faria Vieira(1), Roberto Tetsuo Tanaka(2), Siu Mui Tsai(3), Daniel Vidal Pérez ${ }^{(4)}$ \\ e Célia Maria Maganhotto de Sousa Silva ${ }^{(1)}$
}

\begin{abstract}
(1)Embrapa Meio Ambiente, Caixa Postal 69, CEP 18200-000 Jaguariúna, SP. E-mail: rosana@cnpma.embrapa.br (2)Instituto Agronômico de Campinas, Centro de Grãos e Fibras, Setor de Leguminosas, Av. Theodureto de Almeida Camargo, no 1500, CEP 13075-630 Campinas, SP. E-mail: tanakart@iac.sp.gov.br (3)Universidade de São Paulo, Centro de Energia Nuclear na Agricultura, Caixa Postal 96, CEP 13400-970 Piracicaba, SP. E-mail: tsai@usp.com.br ${ }^{(4)}$ Embrapa Solos, Rua Jardim Botânico, no 1024, CEP 22460-000 Rio de Janeiro, RJ. E-mail: daniel@cpns.embrapa.br
\end{abstract}

\begin{abstract}
Resumo - O objetivo deste trabalho foi verificar o efeito direto e residual da adubação com lodo de esgoto, como fonte de P, na produtividade da soja, na qualidade dos grãos para consumo humano e no potencial de lixiviação do nitrato. O experimento foi realizado no campo e constituiu-se dos seguintes tratamentos: ausência de adubação química e de lodo; adubação química completa; soja com inoculação mais dose zero de lodo; soja com inoculação mais 1,5 t ha-1 de lodo; soja com inoculação mais $3 \mathrm{t} \mathrm{ha}^{-1}$ de lodo; soja com inoculação mais $6 \mathrm{t}$ ha-1 de lodo; e soja com inoculação mais adubação química, exceto a nitrogenada. As maiores produtividades de soja, tanto no primeiro como no segundo ano agrícola, foram obtidas nas duas maiores doses de lodo. Os teores de vários elementos nos grãos de soja, nos tratamentos com lodo, não diferiram, significativamente, daqueles obtidos nos tratamentos testemunha ou com adubação mineral. Perdas de $\mathrm{NO}_{3}{ }^{-}$para o ambiente podem ocorrer, principalmente no período inicial do ciclo da cultura.
\end{abstract}

Termos para indexação: biossólido, fósforo, mineralização de nitrogênio, metais pesados.

\section{Availability of soil nutrients, quality of grains and yield of soybean in soil treated with sewage sludge}

\begin{abstract}
The objective of this work was to evaluate the direct and residual effects of sewage sludge soil application, as a source of phosphorus, on soybean yield, on grain quality for human consumption, and the potential of $\mathrm{NO}_{3}^{-}$leaching. The experiment was carried out in the field, and the following treatments were compared: absence of chemical fertilization and sewage sludge; complete chemical fertilization; inoculated soybean plus dose 0 of sludge; inoculated soybean plus $1.5 \mathrm{t} \mathrm{ha}^{-1}$ of sewage sludge; inoculated soybean plus $3 \mathrm{t} \mathrm{ha}^{-1}$ of sewage sludge; inoculated soybean plus $6 \mathrm{t} \mathrm{ha}^{-1}$ of sewage sludge; and inoculated soybean plus chemical fertilization, except nitrogen. The highest sewage sludge doses resulted in highest soybean yield, in the first and second year. Contents of several elements in soybean grains, under sewage sludge treatments, were not significantly different from those obtained in the control and chemical fertilization treatments. Losses of $\mathrm{NO}_{3}{ }^{-}$to the environment may occur, mainly in the beginning of crop growth cycle.
\end{abstract}

Index terms: biosolid, phosphorus, nitrogen mineralization, heavy metals.

\section{Introdução}

O lodo de esgoto, em conseqüência de sua riqueza em nutrientes, principalmente nitrogênio $(\mathrm{N})$ e fósforo (P), tem sido utilizado em muitos países como fertilizante (Binder et al., 2002). O fósforo é considerado um dos nutrientes limitantes à produção agrícola, em decorrência dos processos de sua adsorção em colóides minerais, amorfos e orgânicos (Novais \& Smith, 1999). Diversos trabalhos demonstraram os benefícios do lodo de esgoto para espécies cultivadas e para algumas ca- racterísticas químicas e físicas do solo (Oliveira et al., 1995; Lindsay \& Logan, 1998). No Brasil, esses trabalhos, porém, se restringem às culturas do sorgo granífero, milheto (Ros et al., 1993), feijão (Vieira et al., 2002) e milho (Berton et al., 1989; Vieira \& Cardoso, 2003). Nenhum trabalho foi realizado com a cultura da soja.

A soja exporta cerca de $150 \mathrm{~kg} \mathrm{ha}^{-1}$ de $\mathrm{N}$ nos grãos, e essa quantidade e aquela contida nos restos culturais podem ser fornecidas, eficientemente, por meio da simbiose com bactérias do gênero Bradyrhizobium. A adição de adubos nitrogenados com a inoculação não 
traz benefícios à eficiência da fixação simbiótica do $\mathrm{N}_{2}$, ao contrário de outros nutrientes como o fósforo e o molibdênio (Giller \& Wilson, 1991). Trabalho realizado com soja, em casa de vegetação, demonstrou que a aplicação equivalente a $23 \mathrm{t} \mathrm{ha}^{-1}$ de lodo de esgoto ao solo (base úmida) supriu as necessidades da planta em $\mathrm{P}$, sem que o $\mathrm{N}$ oriundo do biossólido prejudicasse a fixação simbiótica do $\mathrm{N}_{2}$ (Vieira, 2001). Nesse trabalho, como o lodo de esgoto foi aplicado em plantas com inoculação e sem inoculação, ficou evidente a importância do processo simbiótico na resposta da planta ao biossólido, ou seja, as maiores produtividades foram obtidas pelas plantas que receberam lodo de esgoto, mas que foram submetidas à inoculação.

Alguns elementos normalmente presentes no biossólido ainda são motivo de dúvida quanto aos riscos potenciais de contaminação do meio ambiente, que poderiam advir de sua utilização na agricultura. Em geral, os biossólidos são ricos em $\mathrm{N}$, o que requer um acompanhamento do processo de mineralização desse elemento no solo, uma vez que quantidades excessivas de $\mathrm{NO}_{3}{ }^{-}$poderão, por meio dos processos de lixiviação e desnitrificação, respectivamente, contaminar águas subterrâneas ou provocar o desprendimento de óxido nitroso (Hernández et al., 2002). Ademais, por causa do elevado teor de metais pesados presentes nos lodos, ainda não são conhecidos os danos que poderiam advir do consumo de produtos agrícolas oriundos de locais onde esse insumo é utilizado.

O objetivo deste trabalho foi verificar o efeito direto e residual da adubação com lodo de esgoto, como fonte de $\mathrm{P}$, na produtividade da soja, na qualidade dos grãos para consumo humano e no potencial de lixiviação do nitrato.

\section{Material e Métodos}

O experimento foi iniciado em novembro de 2000, no campo experimental da Embrapa Meio Ambiente, Município de Jaguariúna, SP, em solo classificado como Latossolo Vermelho distroférrico, textura areia/ argilosa (Embrapa, 1999). A composição química inicial $(0-20 \mathrm{~cm})$ do solo foi: $\mathrm{pH}\left(\mathrm{CaCl}_{2}\right), 4,4 ; \mathrm{P}, 8 \mathrm{mg} \mathrm{dm}^{-3}$ (método da resina); $\mathrm{K}^{+}, 1,2 \mathrm{mmol}_{\mathrm{c}} \mathrm{dm}^{-3} ; \mathrm{Ca}^{2+}$, $11 \mathrm{mmol}_{\mathrm{C}} \mathrm{dm}^{-3} ; \mathrm{Mg}^{2+}, 6 \mathrm{mmol}_{\mathrm{C}} \mathrm{dm}^{-3} ; \mathrm{H}+\mathrm{Al}$, $58 \mathrm{mmol}_{\mathrm{C}} \mathrm{dm}^{-3}$; CTC, 76,2 $\mathrm{mmol}_{\mathrm{C}} \mathrm{dm}^{-3} ; \mathrm{V}, 24$ (\%) е MO, $28 \mathrm{~g} \mathrm{dm}^{-3}$ (Embrapa, 1997).

O delineamento experimental foi o de blocos ao acaso, com quatro repetições. Os tratamentos constituíram-se da ausência de adubação química ou lodo de esgoto (testemunha); adubação química completa (AQ); soja com inoculação mais dose zero de lodo de esgoto (I + L0); soja com inoculação mais $1,5 \mathrm{t} \mathrm{ha}^{-1}$ de lodo de esgoto ( I + L1); soja com inoculação mais $3 \mathrm{t} \mathrm{ha}^{-1}$ de lodo de esgoto (I + L2); soja com inoculação mais $6 \mathrm{tha}^{-1} \mathrm{de}$ lodo de esgoto (I + L3) e soja com inoculação mais adubação química (I + AQ), exceto a nitrogenada. Foi aplicado calcário na área experimental, a fim de elevar a saturação por bases a $60 \%$. Nas parcelas do tratamento AQ foram aplicados $\mathrm{P}$ e $\mathrm{K}$, no sulco de semeadura, nas doses de $80 \mathrm{~kg} \mathrm{ha}^{-1}$ de $\mathrm{P}_{2} \mathrm{O}_{5}$ (superfosfato simples) e $60 \mathrm{~kg} \mathrm{ha}^{-1}$ de $\mathrm{K}_{2} \mathrm{O}$ (cloreto de potássio). $\mathrm{O} \mathrm{N}$ foi aplicado na dose de $120 \mathrm{~kg} \mathrm{ha}^{-1}$ de $\mathrm{N}$ (uréia), de forma parcelada, aos 20, 40 e 60 dias da semeadura. Exceto a adubação nitrogenada, as demais foram feitas de acordo com a análise de solo e as recomendações de Mascarenhas \& Tanaka (1996).

As doses de lodo de esgoto foram calculadas com base no teor de P do biossólido e na necessidade da planta nesse nutriente. No cálculo das doses finais de lodo (base seca), foi utilizada a seguinte fórmula: dose de lodo $\left(\mathrm{kg} \mathrm{ha}^{-1}\right)=\mathrm{P}$ recomendado $\left(\mathrm{kg} \mathrm{ha}^{-1}\right) / \mathrm{P}$ contido no lodo de esgoto $\left(\mathrm{kg} \mathrm{kg}^{-1}\right)$. A segunda dose, $3 \mathrm{t} \mathrm{ha}^{-1}$, forneceria a quantidade de P recomendada por Mascarenhas \& Tanaka (1996), ou seja, $80 \mathrm{~kg} \mathrm{ha}^{-1}$ de $\mathrm{P}_{2} \mathrm{O}_{5}$. Por causa do baixo teor de $\mathrm{K}$ do lodo, nas parcelas que receberam 1,5 e $3 \mathrm{t} \mathrm{ha}^{-1}$ do biossólido, aplicou-se cloreto de potássio, para se atingir a dose recomendada de $60 \mathrm{~kg} \mathrm{ha}^{-1}$ de $\mathrm{K}_{2} \mathrm{O}$.

O lodo foi distribuído uniformemente na superfície e incorporado na camada de $0-20 \mathrm{~cm}$ do solo. O biossólido, anaerobicamente digerido, fornecido pela Estação de Tratamento de Lodo de Barueri, SP, que, além de esgotos domésticos recebe esgotos industriais, apresentava as seguintes características químicas: $\mathrm{N}, 40,7 \mathrm{~g} \mathrm{~kg}^{-1}$; $\mathrm{P}, 11,5 \mathrm{~g} \mathrm{~kg}^{-1}$; K, 8,3 $\mathrm{g} \mathrm{kg}^{-1}$; Ca, $17 \mathrm{~g} \mathrm{~kg}^{-1}$; Mg, 2,5 g kg-1; S, 11,7 $\mathrm{g} \mathrm{kg}^{-1}$; B, 9 mg kg-1; $\mathrm{Cu}, 707 \mathrm{mg} \mathrm{kg}^{-1}$; Fe, 22,9 $\mathrm{g} \mathrm{kg}^{-1}$; Mn, $168 \mathrm{mg} \mathrm{kg}^{-1}$; Zn, 2.314 mg kg$^{-1}$; Al, 9,51 g kg-1$^{-1}$ Cd, 9,2 g kg k$^{-1}$; Pb, $151 \mathrm{mg} \mathrm{kg}^{-1}$; Cr, $597 \mathrm{mg} \mathrm{kg}^{-1}$; Ni, $268 \mathrm{mg} \mathrm{kg}^{-1}$; Na, $4 \mathrm{~g} \mathrm{~kg}^{-1}$; umidade, $767 \mathrm{~g} \mathrm{~kg}^{-1}$.

A área experimental era de $2.610 \mathrm{~m}^{2}$, formada de quatro blocos de $290 \mathrm{~m}^{2}$. Cada parcela experimental $\left(20 \mathrm{~m}^{2}\right)$ foi constituída por oito sulcos de semeadura, de $5 \mathrm{~m}$ de comprimento, espaçados entre si de $0,5 \mathrm{~m}$, nos quais foram semeadas 20 sementes por metro. As duas linhas externas foram utilizadas como bordaduras, e as duas centrais, para obtenção da produtividade. As outras linhas foram usadas nas colheitas periódicas de plantas. 
Na primeira semeadura, foi usado inoculante oleoso nas sementes (cv. IAC 22), conforme a recomendação do fabricante.

No primeiro cultivo, para avaliação do potencial de lixiviação do $\mathrm{NO}_{3}{ }^{-}$, foram iniciadas as coletas mas camadas de solo aos 28 dias após a emergência das plantas (DAE), e repetidas a cada 21 dias, até o 146o DAE. As amostras foram coletadas nas camadas de $0-20 \mathrm{~cm}$ (em todas as coletas), 20-40 e 40-60 cm (na primeira e última coletas), e analisadas imediatamente em laboratório, quanto aos teores de $\mathrm{NH}_{4}{ }^{+}$e de $\mathrm{NO}_{3}{ }^{-}$(Tedesco et al., 1985). Na determinação dos teores de P (método da resina), nas diferentes parcelas, as amostras de solo foram coletadas na camada de $0-20 \mathrm{~cm}$ aos 69 e 85 DAE.

Os teores de $\mathrm{N}$ total, acumulado na parte aérea das plantas de soja, foram avaliados aos 32 e 64 DAE, em quatro plantas de cada parcela (Tedesco et al., 1985). No final do ciclo, após a determinação da produtividade, os grãos foram armazenados a $5^{\circ} \mathrm{C}$, para determinação posterior dos teores de $\mathrm{Fe}, \mathrm{Zn}, \mathrm{Cu}, \mathrm{Cd}, \mathrm{Pb}$ e $\mathrm{Ni}$ (Carmo et al., 2000).

No segundo ano de cultivo da soja, não foi aplicado lodo de esgoto ao solo, uma vez que se procurou avaliar o efeito residual daquele aplicado no ano anterior. Antes da semeadura foram coletadas amostras de solo na camada de $0-20 \mathrm{~cm}$ em todos os tratamentos, para análise dos seguintes atributos químicos: $\mathrm{pH}, \mathrm{P}, \mathrm{K}^{+}, \mathrm{Ca}^{2+}, \mathrm{Mg}^{2+}$, B, Cu, Fe, Mn, Zn, H+Al, SB (soma de bases), CTC (capacidade de troca de cátions) e V (saturação por bases). Em cada tratamento, para cada repetição, foram coletadas 4 subamostras de solo, que foram homogeneizadas, de modo a se obter uma amostra composta de 16 subamostras por tratamento.

As adubações químicas, referentes aos tratamentos AQ e I + AQ, utilizadas no primeiro cultivo, foram repetidas no segundo. $\mathrm{O}$ inoculante utilizado, à base de turfa, foi produzido no Centro de Energia Nuclear na Agricultura, USP, Piracicaba, SP.

As coletas das amostras, para as análises de $\mathrm{N}$ mineral no solo, iniciaram-se aos 18 DAE e foram repetidas a cada 15 dias até o 95o DAE. A técnica dos tubos de PVC foi utilizada no segundo cultivo. Para tanto, as coletas das amostras de solo foram feitas conforme Dou et al. (1997), com modificações (Vieira \& Cardoso, 2003). Em cada parcela, foram colocados quatro pares de colunas de PVC (5 cm de diâmetro, 22 cm de altura), distribuídas aleatoriamente nas entrelinhas da soja, na camada de $0-20 \mathrm{~cm}$. Nas parcelas do tratamento AQ, as colunas foram colocadas o mais próximo possível da linha de aplicação da uréia. Um tubo de cada par foi coberto com tampa de PVC de $10 \mathrm{~cm}$ de diâmetro, com a finalidade de se evitar a lixiviação do $\mathrm{NO}_{3}{ }^{-}$em períodos de chuva. A quantidade de nitrato lixiviado foi quantificada pela diferença entre os teores desse ânion nos dois sistemas (aberto e fechado). Os solos das colunas foram coletados em períodos definidos, e novas colunas foram imediatamente recolocadas em locais escolhidos aleatoriamente, mas nunca a menos de $1 \mathrm{~m}$ das bordaduras. Em cada parcela, as porções do mesmo sistema (tampado e não tampado) foram misturadas e homogeneizadas, de modo a se obter quatro amostras compostas por tratamento, as quais foram analisadas e processadas conforme descrito para o primeiro cultivo.

Os teores de $\mathrm{N}$ total na parte aérea e os de $\mathrm{P}$ no solo foram quantificados aos 32 e 71 DAE, conforme métodos usados no primeiro cultivo. Após a colheita, os grãos foram submetidos às mesmas análises do primeiro cultivo.

Os dados de precipitação pluvial foram obtidos na estação meteorológica localizada na Embrapa Meio Ambiente, Município de Jaguariúna, SP.

O programa ANOVA foi utilizado na análise estatística dos dados e aplicação do teste F. O teste de Duncan foi utilizado na comparação das médias dos tratamentos a $5 \%$ de probabilidade.

\section{Resultados e Discussão}

Os teores de P no solo, no primeiro ano de cultivo, na primeira avaliação, foram maiores nos tratamentos com lodo; não houve, porém, diferença significativa entre as doses do composto (Tabela 1). Aos 85 DAE, verificou-se decréscimo desse nutriente no solo de todos os tratamentos, em relação à avaliação anterior; nessa época, verificou-se tendência à maior concentração de $\mathrm{P}$ no solo, com o aumento da dose de lodo. No segundo cultivo, aos 32 DAE não houve diferença significativa entre os teores de $\mathrm{P}$ no solo, entre os tratamentos. Aos 71 DAE, embora sem diferença significativa, verificou-se tendência à menor concentração de $\mathrm{P}$ no solo, no tratamento testemunha e no tratamento com inoculação que não recebeu adubação química nem lodo de esgoto.

A análise de solo realizada antes da semeadura da soja, em 2001 (segundo ano de cultivo), demonstrou que os teores de P nos solos suplementados com lodo fo- 
ram em média $23 \%$ e $45 \%$ maiores, respectivamente, do que a média dos tratamentos AQ + (I + AQ) e a média dos tratamentos testemunha $+(I+L 0)$. Segundo Mazzarino et al. (1998), a liberação de P do biossólido depende, primeiramente, do tipo de solo e, em segundo lugar, da origem do composto, ao contrário do que ocorre com o nitrogênio. Houve, também, um aumento dos teores de $\mathrm{Cu}$ e Zn nos solos suplementados com lodo. Quanto ao $\mathrm{Zn}$, seu aumento relacionou-se positivamente com as doses de lodo (Tabela 2), o que corrobora os dados de White et al. (1997).

Tabela 1. Teores de fósforo no solo cultivado com soja por dois anos agrícolas, em resposta à adubação com lodo de esgoto. 2000/2001 (efeito direto) e 2001/2002 (efeito residual) ${ }^{(1)}$.

\begin{tabular}{|c|c|c|c|c|}
\hline \multirow[t]{2}{*}{ Tratamento $^{(2)}$} & \multicolumn{2}{|c|}{ Ano $2000 / 2001$} & \multicolumn{2}{|c|}{ Ano $2001 / 2002$} \\
\hline & $69 \mathrm{DAE}$ & $85 \mathrm{DAE}$ & $32 \mathrm{DAE}$ & $71 \mathrm{DAE}$ \\
\hline & ------------------. & $(\mathrm{m}$ & ------ & -------- \\
\hline Testemunha & $8,2 b$ & $5,5 \mathrm{~b}$ & $9,0 \mathrm{a}$ & $8,5 \mathrm{a}$ \\
\hline $\mathrm{AQ}$ & $11,0 \mathrm{ab}$ & $5,5 b$ & $13,0 \mathrm{a}$ & $12,0 \mathrm{a}$ \\
\hline $\mathrm{I}+\mathrm{L} 0$ & $8,5 \mathrm{~b}$ & $5,5 b$ & $10,0 \mathrm{a}$ & $8,5 \mathrm{a}$ \\
\hline $\mathrm{I}+\mathrm{L} 1$ & $13,5 \mathrm{a}$ & $7,3 b$ & $10,0 \mathrm{a}$ & $9,5 \mathrm{a}$ \\
\hline $\mathrm{I}+\mathrm{L} 2$ & $13,0 \mathrm{a}$ & $8,0 \mathrm{~b}$ & $11,0 \mathrm{a}$ & $9,5 \mathrm{a}$ \\
\hline $\mathrm{I}+\mathrm{L} 3$ & $14,5 \mathrm{a}$ & $12,0 \mathrm{ab}$ & $10,0 \mathrm{a}$ & $10,0 \mathrm{a}$ \\
\hline $\mathrm{I}+\mathrm{AQ}$ & $9,0 \mathrm{~b}$ & $5,8 \mathrm{~b}$ & $13,0 \mathrm{a}$ & $12,0 \mathrm{a}$ \\
\hline
\end{tabular}

${ }^{(1)}$ Médias seguidas pela mesma letra, na coluna, dentro de cada época de avaliação, não diferem entre si pelo teste de Duncan a $5 \%$ de probabilidade. (2) AQ: adubação química completa $\left(60 \mathrm{~kg} \mathrm{ha}^{-1}\right.$ de $\mathrm{P}_{2} \mathrm{O}_{5}$, $60 \mathrm{~kg} \mathrm{ha}^{-1}$ de $\mathrm{K}_{2} \mathrm{O}, 120 \mathrm{~kg} \mathrm{ha}^{-1}$ de N); I + L0: soja com inoculação, sem aplicação de lodo de esgoto; I + L1: soja com inoculação, mais $1,5 \mathrm{t} \mathrm{ha}^{-1}$ de lodo de esgoto; I + L2: soja com inoculação, mais 3 t ha-1 de lodo de esgoto; I + L3: soja com inoculação, mais $6 \mathrm{t} \mathrm{ha}^{-1}$ de lodo de esgoto; I + AQ: soja com inoculação, mais adubação química, exceto a nitrogenada.
Aos 28 DAE, no primeiro cultivo, os teores de $\mathrm{N}$ mineral foram maiores nos tratamentos AQ, I + L2 e I + L3, na camada de $0-20 \mathrm{~cm}$ de solo (Tabela 3). Nesses tratamentos, os teores de 39, 28 e $29,5 \mathrm{~kg} \mathrm{ha}^{-1}$ de $\mathrm{N}$ mineral foram $83 \%$, $76 \%$ e $78 \%$ superiores, respectivamente, à média dos tratamentos que não receberam fertilizante nitrogenado (testemunha, $\mathrm{I}+\mathrm{L0}$ e I + AQ). O alto teor de $\mathrm{N}$ no tratamento AQ pode estar associado à presença de grânulos de adubo no solo coletado. Houve também acréscimo dos teores de $\mathrm{N}$ mineral, na maior dose de lodo, com o aumento da profundidade do solo. Nas camadas do solo de $20-40 \mathrm{~cm}$ e 40-60 cm, o tratamento I + L3 apresentou, respectivamente, $50 \%$ e $24 \%$ mais $\mathrm{N}$ mineral do que a média dos outros tratamentos. Aos 49, 70 e 91 DAE, os teores de $\mathrm{N}$ mineral diminuíram em alguns tratamentos, ou mantiveram-se semelhantes àqueles observados aos 28 DAE, na camada de $0-20 \mathrm{~cm}$ de solo. Aos 146 DAE, no tratamento I + L3, na maior profundidade de solo, o teor de $\mathrm{N}$ mineral foi $48 \%$ maior do que a média dos tratamentos que não receberam adubo nitrogenado por meio do lodo ou do fertilizante (testemunha, I + L0 e $\mathrm{I}+\mathrm{AQ}$ ). Os resultados individuais do $\mathrm{NH}_{4}{ }^{+}$e do $\mathrm{NO}_{3}{ }^{-}$ demonstraram uma intensa nitrificação do solo.

Aos 18 e 33 DAE, no segundo cultivo, os teores de N no solo aumentaram com o aumento das doses de lodo. Nas avaliações realizadas após os 50 DAE, no segundo cultivo, os teores de $\mathrm{N}$ mineral no solo foram maiores do que os obtidos no primeiro cultivo, independentemente do tratamento (Tabela 4). A maior precipitação pluvial

Tabela 2. Análise química dos solos dos tratamentos, antes do segundo cultivo da soja, no ano agrícola 2001/2002(1).

\begin{tabular}{|c|c|c|c|c|c|c|c|}
\hline \multirow[t]{2}{*}{ Atributos químicos } & \multicolumn{7}{|c|}{ Tratamentos $^{(2)}$} \\
\hline & Testemunha & $\mathrm{AQ}$ & $\mathrm{I}+\mathrm{L} 0$ & $\mathrm{I}+\mathrm{L} 1$ & $\mathrm{I}+\mathrm{L} 2$ & $\mathrm{I}+\mathrm{L3}$ & $\mathrm{I}+\mathrm{AQ}$ \\
\hline $\mathrm{pH}$ & 5,30 & 5,38 & 5,38 & 5,34 & 5,32 & 5,28 & 5,32 \\
\hline $\mathrm{P}\left(\mathrm{mg} \mathrm{dm}^{-3}\right)$ & 4,40 & 5,75 & 4,60 & 9,20 & 6,40 & 7,40 & 6,80 \\
\hline $\mathrm{K}\left(\mathrm{mmol}_{\mathrm{c}} \mathrm{dm}^{-3}\right)$ & 0,70 & 0,98 & 0,86 & 0,92 & 1,00 & 0,88 & 0,94 \\
\hline $\mathrm{Ca}\left(\mathrm{mmol}_{\mathrm{c}} \mathrm{dm}^{-3}\right)$ & 20,40 & 20,80 & 22,40 & 19,40 & 23,20 & 22,20 & 24,00 \\
\hline $\mathrm{Mg}\left(\mathrm{mmol}_{\mathrm{c}} \mathrm{dm}^{-3}\right)$ & 16,20 & 15,20 & 14,75 & 15,20 & 19,00 & 16,60 & 19,00 \\
\hline $\mathrm{H}+\mathrm{Al}\left(\mathrm{mmol}_{\mathrm{c}} \mathrm{dm}^{-3}\right)$ & 32,60 & 30,40 & 32,00 & 31,00 & 31,20 & 32,40 & 29,75 \\
\hline $\mathrm{SB}\left(\mathrm{mmol}_{\mathrm{c}} \mathrm{dm}^{-3}\right)$ & 29,92 & 37,00 & 35,10 & 35,50 & 43,20 & 39,68 & 40,40 \\
\hline CTC $\left(\mathrm{mmol}_{\mathrm{c}} \mathrm{dm}^{-3}\right)$ & 69,92 & 67,54 & 73,10 & 66,66 & 74,30 & 72,18 & 70,44 \\
\hline $\mathrm{V}(\%)$ & 53,60 & 54,80 & 55,20 & 53,20 & 57,60 & 54,80 & 60,00 \\
\hline $\mathrm{B}\left(\mathrm{mg} \mathrm{kg}^{-1}\right)$ & 0,15 & 0,15 & 0,16 & 0,16 & 0,17 & 0,17 & 0,16 \\
\hline $\mathrm{Cu}\left(\mathrm{mg} \mathrm{kg}^{-1}\right)$ & 0,70 & 0,72 & 0,76 & 0,90 & 0,86 & 1,00 & 0,76 \\
\hline $\mathrm{Fe}\left(\mathrm{mg} \mathrm{kg}^{-1}\right)$ & 34,60 & 36,60 & 39,40 & 39,60 & 39,40 & 42,80 & 38,60 \\
\hline $\operatorname{Mn}\left(\mathrm{mg} \mathrm{kg}{ }^{-1}\right)$ & 1,58 & 1,74 & 1,66 & 1,62 & 1,46 & 1,78 & 1,58 \\
\hline $\mathrm{Zn}\left(\mathrm{mg} \mathrm{kg}^{-1}\right)$ & 0,66 & 0,70 & 0,82 & 1,18 & 1,48 & 1,60 & 1,00 \\
\hline
\end{tabular}

(1)Valores obtidos de uma amostra composta de 16 subamostras, por tratamento. (2)AQ: adubação química completa (60 kg ha-1 de $\mathrm{P}_{2} \mathrm{O}_{5}$, 60 kg ha ${ }^{-1}$ de $\mathrm{K}_{2} \mathrm{O}, 120$ kg ha-1 de N); I + L0: soja com inoculação, sem aplicação de lodo de esgoto; I + L1: soja com inoculação, mais 1,5 t ha-1 de lodo de esgoto; I + L2: soja com inoculação, mais 3 t ha $^{-1}$ de lodo de esgoto; I + L3: soja com inoculação, mais 6 t ha ${ }^{-1}$ de lodo de esgoto; I + AQ: soja com inoculação, mais adubação química, exceto a nitrogenada. 
nesse ano agrícola, em relação à observada no ano agrícola de 2000/2001, pode ter sido responsável pela maior mineralização do N orgânico do solo. Em janeiro de 2002, a precipitação pluvial foi de $341 \mathrm{~mm}$, enquanto nesse mesmo período, no ano de 2001, ela foi de $119 \mathrm{~mm}$.

Nas duas primeiras avaliações, realizadas aos 18 DAE e aos 33 DAE, respectivamente, nos dias 3 e 18 de janeiro, os menores teores de $\mathrm{N}$ mineral total encontrados no solo podem ser conseqüência da escassez de chuvas, pois $67 \%$ delas ocorreram após 19 de janeiro. Como não houve diferença significativa entre os teores de $\mathrm{N}$ da testemunha e dos tratamentos com lodo, o $\mathrm{N}$ mineral encontrado foi, provavelmente, oriundo da mineralização do N orgânico do solo. Perdas de $\mathrm{N}$ por lixiviação não foram detectadas, nem mesmo nos períodos de intensa precipitação pluvial.

Os teores de $\mathrm{N}$ no solo, no primeiro cultivo da soja, demonstraram que, no tratamento I + L3, a quantidade aplicada do biossólido pode ter ocasionado perdas de $\mathrm{N}$ por lixiviação. Aos 28 DAE foram encontrados $34 \mathrm{~kg} \mathrm{ha}^{-1} \mathrm{de}^{\mathrm{NO}_{3}}{ }^{-}$na camada de $40-60 \mathrm{~cm}$. Vieira
\& Cardoso (2003), Mazzarino et al. (1998) e Hernández et al. (2002) constataram que, logo após a adição do lodo ao solo, ocorre intenso processo de mineralização do N orgânico do lodo. Como as medições no solo cultivado com soja tiveram início somente aos $28 \mathrm{DAE}$, as perdas potenciais de $\mathrm{N}$ nesse solo podem ter sido subestimadas.

No tratamento I + L2 ocorreu tendência à maior quantidade de nitrato na maior profundidade. Barbarick et al. (1996) também relataram perdas de $\mathrm{NO}_{3}{ }^{-}$por lixiviação, além da zona das raízes, em solo tratado com lodo de esgoto.

Aos 32 DAE do primeiro cultivo, a quantidade de $\mathrm{N}$ acumulada na parte aérea foi maior nos tratamentos que receberam lodo de esgoto, contrariamente ao ocorrido no ano agrícola seguinte (Tabela 5). Aos 64 DAE do primeiro cultivo, as maiores quantidades acumuladas de $\mathrm{N}$ na parte aérea foram obtidas nos tratamentos AQ e nos tratamentos com lodo. Aos $71 \mathrm{DAE}$ do segundo cultivo, houve aumento da quantidade acumulada de $\mathrm{N}$ na planta com as doses de lodo; os maiores valores des-

Tabela 3. Nitrogênio mineral em camadas de solo submetido a tratamentos com adubos minerais e lodo de esgoto, aos 28, 49, 70, 91 e 146 dias após a emergência (DAE) da soja, em 2000/2001(efeito direto) ${ }^{(1)}$.

\begin{tabular}{|c|c|c|c|c|c|c|c|c|c|}
\hline \multirow[t]{2}{*}{ Tratamento $^{(2)}$} & \multicolumn{3}{|c|}{$28 \mathrm{DAE}$} & \multirow{2}{*}{$\begin{array}{l}49 \mathrm{DAE} \\
0-20 \mathrm{~cm}\end{array}$} & \multirow{2}{*}{$\begin{array}{l}70 \mathrm{DAE} \\
0-20 \mathrm{~cm}\end{array}$} & \multirow{2}{*}{$\begin{array}{l}91 \mathrm{DAE} \\
0-20 \mathrm{~cm} \\
\end{array}$} & \multicolumn{3}{|c|}{$146 \mathrm{DAE}$} \\
\hline & $0-20 \mathrm{~cm}$ & $20-40 \mathrm{~cm}$ & $40-60 \mathrm{~cm}$ & & & & $0-20 \mathrm{~cm}$ & $20-40 \mathrm{~cm}$ & $40-60 \mathrm{~cm}$ \\
\hline & \multicolumn{9}{|c|}{$\mathrm{NH}_{4}^{+}+\mathrm{NO}_{3}^{-}\left(\mu \mathrm{g} \mathrm{g}^{-1}\right.$ de solo $)$} \\
\hline Testemunha & $7,0 \mathrm{~b}$ & $7,0 \mathrm{~b}$ & $14,0 \mathrm{ab}$ & $6,8 \mathrm{~b}$ & $4,5 \mathrm{a}$ & $8,2 \mathrm{a}$ & $7,5 \mathrm{c}$ & $11,8 \mathrm{a}$ & $3,8 \mathrm{~d}$ \\
\hline $\mathrm{AQ}$ & $19,5 \mathrm{a}$ & $10,2 b$ & $10,5 b$ & $12,2 \mathrm{a}$ & $7,0 \mathrm{a}$ & $9,0 \mathrm{a}$ & $8,2 \mathrm{bc}$ & $10,8 \mathrm{a}$ & $4,8 \mathrm{~cd}$ \\
\hline $\mathrm{I}+\mathrm{L} 0$ & $6,5 b$ & $10,2 b$ & $13,2 \mathrm{ab}$ & $7,0 \mathrm{~b}$ & $4,0 \mathrm{a}$ & $6,5 \mathrm{a}$ & $8,0 \mathrm{bc}$ & $11,8 \mathrm{a}$ & 7,2abcd \\
\hline $\mathrm{I}+\mathrm{L1}$ & $7,2 \mathrm{~b}$ & $8,0 \mathrm{~b}$ & $14,0 \mathrm{ab}$ & $5,2 b$ & $4,8 \mathrm{a}$ & $5,5 \mathrm{a}$ & $9,5 \mathrm{abc}$ & $10,0 \mathrm{a}$ & $8,2 \mathrm{abcd}$ \\
\hline $\mathrm{I}+\mathrm{L} 2$ & $14,0 \mathrm{ab}$ & $11,0 \mathrm{~b}$ & $14,8 \mathrm{ab}$ & $4,5 b$ & $4,5 \mathrm{a}$ & $5,2 \mathrm{a}$ & $13,2 \mathrm{a}$ & $11,8 \mathrm{a}$ & $9,5 \mathrm{ab}$ \\
\hline $\mathrm{I}+\mathrm{L} 3$ & $14,8 \mathrm{ab}$ & $18,0 \mathrm{a}$ & $17,5 \mathrm{a}$ & $6,5 b$ & $5,0 \mathrm{a}$ & $5,5 \mathrm{a}$ & $12,0 \mathrm{ab}$ & $9,2 \mathrm{a}$ & $10,8 \mathrm{a}$ \\
\hline \multirow[t]{2}{*}{$\mathrm{I}+\mathrm{AQ}$} & $6,2 \mathrm{~b}$ & $7,5 \mathrm{~b}$ & $-^{(3)}$ & $4,0 \mathrm{~b}$ & $8,0 \mathrm{a}$ & $7,2 \mathrm{a}$ & $6,5 \mathrm{c}$ & $10,8 \mathrm{a}$ & $5,8 \mathrm{bcd}$ \\
\hline & \multicolumn{9}{|c|}{$\mathrm{NH}_{4}^{+}\left(\mu \mathrm{g} \mathrm{g}^{-1}\right.$ de solo $)$} \\
\hline Testemunha & $1,0 \mathrm{~b}$ & $1,8 \mathrm{a}$ & $1,2 \mathrm{ab}$ & $3,0 \mathrm{~b}$ & $2,2 \mathrm{a}$ & $3,8 \mathrm{ab}$ & $2,5 \mathrm{a}$ & $5,5 \mathrm{a}$ & $1,8 \mathrm{~d}$ \\
\hline $\mathrm{AQ}$ & $12,8 \mathrm{a}$ & $5,0 \mathrm{a}$ & $0,5 \mathrm{ab}$ & $6,0 \mathrm{a}$ & $3,5 \mathrm{a}$ & $4,0 \mathrm{a}$ & $2,2 \mathrm{a}$ & $5,8 \mathrm{a}$ & $2,2 \mathrm{~cd}$ \\
\hline $\mathrm{I}+\mathrm{L} 0$ & $1,5 b$ & $4,5 \mathrm{a}$ & $1,8 \mathrm{ab}$ & $3,0 \mathrm{~b}$ & $3,0 \mathrm{a}$ & $4,2 \mathrm{a}$ & $2,0 \mathrm{a}$ & $6,2 \mathrm{a}$ & 3,5abcd \\
\hline $\mathrm{I}+\mathrm{L} 1$ & $1,5 b$ & $3,0 \mathrm{a}$ & $2,2 \mathrm{a}$ & $3,0 \mathrm{~b}$ & $3,5 \mathrm{a}$ & $3,5 \mathrm{ab}$ & $3,0 \mathrm{a}$ & $4,5 \mathrm{a}$ & $5,2 \mathrm{ab}$ \\
\hline $\mathrm{I}+\mathrm{L} 2$ & $1,8 \mathrm{~b}$ & $4,2 \mathrm{a}$ & $0,0 \mathrm{~b}$ & $2,0 \mathrm{~b}$ & $2,8 \mathrm{a}$ & $3,5 \mathrm{ab}$ & $4,0 \mathrm{a}$ & $5,5 \mathrm{a}$ & $4,8 \mathrm{abc}$ \\
\hline $\mathrm{I}+\mathrm{L} 3$ & $1,8 \mathrm{~b}$ & $4,2 \mathrm{a}$ & $0,5 \mathrm{ab}$ & $3,2 b$ & $3,0 \mathrm{a}$ & $2,0 \mathrm{~b}$ & $3,0 \mathrm{a}$ & $4,0 \mathrm{a}$ & $6,0 \mathrm{a}$ \\
\hline \multirow[t]{2}{*}{$\mathrm{I}+\mathrm{AQ}$} & $1,5 \mathrm{~b}$ & $2,8 \mathrm{a}$ & $-(3)$ & $2,5 b$ & $5,0 \mathrm{a}$ & $3,8 \mathrm{ab}$ & $1,8 \mathrm{a}$ & $6,0 \mathrm{a}$ & $3,0 \mathrm{bcd}$ \\
\hline & \multicolumn{9}{|c|}{$\mathrm{NO}_{3}^{-}\left(\mu \mathrm{g} \mathrm{g}^{-1}\right.$ de solo $)$} \\
\hline Testemunha & $6,0 \mathrm{a}$ & $5,2 b$ & $12,8 b c$ & $3,8 \mathrm{ab}$ & $2,2 \mathrm{a}$ & $4,5 \mathrm{a}$ & $5,0 \mathrm{~b}$ & $6,2 \mathrm{a}$ & $2,0 \mathrm{~b}$ \\
\hline $\mathrm{AQ}$ & $6,8 \mathrm{a}$ & $5,2 b$ & $10,0 \mathrm{c}$ & $6,2 \mathrm{a}$ & $3,5 \mathrm{a}$ & $5,0 \mathrm{a}$ & $6,0 \mathrm{ab}$ & $5,0 \mathrm{a}$ & $2,5 \mathrm{ab}$ \\
\hline $\mathrm{I}+\mathrm{L} 0$ & $5,0 \mathrm{a}$ & $5,8 \mathrm{~b}$ & $11,5 b c$ & $4,0 \mathrm{ab}$ & $1,0 \mathrm{a}$ & $2,2 \mathrm{a}$ & $6,0 \mathrm{ab}$ & $5,5 \mathrm{a}$ & $3,8 \mathrm{ab}$ \\
\hline $\mathrm{I}+\mathrm{L} 1$ & $5,8 \mathrm{a}$ & $5,0 \mathrm{~b}$ & $11,8 b c$ & $2,2 b$ & $1,2 \mathrm{a}$ & $2,0 \mathrm{a}$ & $6,5 \mathrm{ab}$ & $5,5 \mathrm{a}$ & $3,0 \mathrm{ab}$ \\
\hline $\mathrm{I}+\mathrm{L} 2$ & $12,2 \mathrm{a}$ & $6,8 \mathrm{~b}$ & $14,8 \mathrm{ab}$ & $2,5 b$ & $1,8 \mathrm{a}$ & $1,8 \mathrm{a}$ & $9,2 \mathrm{a}$ & $6,2 \mathrm{a}$ & $4,8 \mathrm{a}$ \\
\hline $\mathrm{I}+\mathrm{L} 3$ & $13,0 \mathrm{a}$ & $13,8 \mathrm{a}$ & $17,0 \mathrm{a}$ & $3,2 b$ & $2,0 \mathrm{a}$ & $3,5 \mathrm{a}$ & $9,0 \mathrm{a}$ & $5,2 \mathrm{a}$ & $4,8 \mathrm{a}$ \\
\hline $\mathrm{I}+\mathrm{AQ}$ & $4,8 \mathrm{a}$ & $4,8 \mathrm{~b}$ & $-(3)$ & $1,5 \mathrm{~b}$ & $3,0 \mathrm{a}$ & $3,5 \mathrm{a}$ & $4,8 \mathrm{~b}$ & $4,8 \mathrm{a}$ & $2,8 \mathrm{ab}$ \\
\hline
\end{tabular}

${ }^{(1)}$ Médias seguidas pela mesma letra, na coluna, dentro de cada época de avaliação, não diferem entre si pelo teste de Duncan a $5 \%$ de probabilidade.

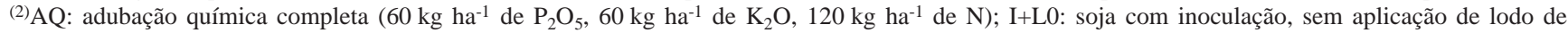
esgoto; I + L1: soja com inoculação, mais 1,5 t ha-1 de lodo de esgoto; I + L2: soja com inoculação, mais 3 t ha-1 de lodo de esgoto; I + L3: soja com inoculação mais $6 \mathrm{t} \mathrm{ha}^{-1}$ de lodo de esgoto; I + AQ: soja com inoculação, mais adubação química, exceto a nitrogenada. (3)Dados variáveis. 
se elemento na parte aérea foram obtidos nos tratamentos AQ, I + L2 e I + L3 (Tabela 5).

No segundo cultivo, apesar dos teores de $\mathrm{N}$ no solo não diferirem significativamente entre os tratamentos, as quantidades acumuladas de $\mathrm{N}$ na parte aérea foram maiores nos tratamentos com lodo. Tal fato pode estar associado à maior eficiência do processo de fixação do $\mathrm{N}$ atmosférico pelas plantas das parcelas suplementadas com o biossólido (Vieira, 2001).

O lodo de esgoto pode conter numerosos componentes requeridos para o crescimento microbiano, o que pode aumentar a atividade de microrganismos do solo, inclusive os rizóbios (Angle et al., 1992; Abd-Alla \& Yan, 1999). É importante salientar que, no primeiro ano de cultivo, não ocorreu formação de nódulos nas raízes da soja. Após a semeadura da soja, que não foi irrigada, ocorreu período de estiagem com temperaturas elevadas que podem ter afetado a viabilidade dos rizóbios, tendo pre-

Tabela 4. Nitrogênio mineral do solo cultivado com soja e submetido a tratamentos com adubos minerais e lodo de esgoto, em 2001/2002 (efeito residual) ${ }^{(1)}$.

\begin{tabular}{|c|c|c|c|c|c|c|}
\hline \multirow[t]{2}{*}{ Tratamento $^{(2)}$} & \multicolumn{6}{|c|}{ Dias após a emergência (DAE) } \\
\hline & 18 & 33 & 50 & 65 & 80 & 95 \\
\hline & \multicolumn{6}{|c|}{$\mathrm{NH}_{4}^{+}+\mathrm{NO}_{3}^{-}\left(\mu \mathrm{g} \mathrm{g}^{-1}\right.$ de solo $)$} \\
\hline Testemunha & $9,0 \mathrm{bc}$ & $8,5 \mathrm{ab}$ & $-(3)$ & $39,5 \mathrm{a}$ & $28,8 \mathrm{a}$ & $20,0 \mathrm{a}$ \\
\hline AQ & $11,5 \mathrm{a}$ & - & - & $40,5 \mathrm{a}$ & $28,4 \mathrm{a}$ & $19,8 \mathrm{a}$ \\
\hline $\mathrm{I}+\mathrm{L} 0$ & $7,0 \mathrm{c}$ & $9,2 \mathrm{ab}$ & - & $40,5 \mathrm{a}$ & $26,4 \mathrm{a}$ & $12,8 \mathrm{~b}$ \\
\hline $\mathrm{I}+\mathrm{L} 1$ & $8,8 \mathrm{bc}$ & $8,0 \mathrm{~b}$ & - & $43,2 \mathrm{a}$ & $29,6 \mathrm{a}$ & $15,8 \mathrm{ab}$ \\
\hline $\mathrm{I}+\mathrm{L} 2$ & $9,0 \mathrm{bc}$ & $9,0 \mathrm{ab}$ & - & $44,0 \mathrm{a}$ & $24,0 \mathrm{a}$ & $15,0 \mathrm{ab}$ \\
\hline $\mathrm{I}+\mathrm{L} 3$ & $10,0 \mathrm{ab}$ & $12,2 \mathrm{a}$ & - & $40,2 \mathrm{a}$ & $34,2 \mathrm{a}$ & $20,0 \mathrm{a}$ \\
\hline \multirow[t]{2}{*}{$\mathrm{I}+\mathrm{AQ}$} & $8,2 \mathrm{bc}$ & $6,2 \mathrm{~b}$ & - & $41,2 \mathrm{a}$ & $30,5 \mathrm{a}$ & $14,2 \mathrm{ab}$ \\
\hline & \multicolumn{6}{|c|}{$\mathrm{NH}_{4}^{+}\left(\mu \mathrm{g} \mathrm{g}^{-1}\right.$ de solo $)$} \\
\hline Testemunha & $5,2 \mathrm{ab}$ & $5,2 \mathrm{a}$ & - & $31,2 \mathrm{a}$ & $15,8 \mathrm{a}$ & $10,5 \mathrm{a}$ \\
\hline $\mathrm{AQ}$ & $6,0 \mathrm{a}$ & - & - & $32,8 \mathrm{a}$ & $14,4 \mathrm{a}$ & $9,0 \mathrm{a}$ \\
\hline $\mathrm{I}+\mathrm{L} 0$ & $4,2 b$ & $5,5 \mathrm{a}$ & - & $32,0 \mathrm{a}$ & $14,4 \mathrm{a}$ & $6,5 \mathrm{a}$ \\
\hline $\mathrm{I}+\mathrm{L} 1$ & $5,2 \mathrm{ab}$ & $4,5 \mathrm{a}$ & - & $35,0 \mathrm{a}$ & $14,0 \mathrm{a}$ & $7,5 \mathrm{a}$ \\
\hline $\mathrm{I}+\mathrm{L} 2$ & $5,0 \mathrm{ab}$ & $4,5 \mathrm{a}$ & - & $35,5 \mathrm{a}$ & $11,8 \mathrm{a}$ & $7,0 \mathrm{a}$ \\
\hline $\mathrm{I}+\mathrm{L} 3$ & $6,0 \mathrm{a}$ & $4,5 \mathrm{a}$ & - & $33,0 \mathrm{a}$ & $14,6 \mathrm{a}$ & $9,2 \mathrm{a}$ \\
\hline \multirow[t]{2}{*}{$\mathrm{I}+\mathrm{AQ}$} & $4,8 \mathrm{ab}$ & $3,8 \mathrm{a}$ & - & $32,2 \mathrm{a}$ & $13,0 \mathrm{a}$ & $6,8 \mathrm{a}$ \\
\hline & \multicolumn{6}{|c|}{$\mathrm{NO}_{3}^{-}\left(\mu \mathrm{g} \mathrm{g}^{-1}\right.$ de solo $)$} \\
\hline Testemunha & $3,8 b$ & $3,2 b$ & - & $8,2 \mathrm{a}$ & $13,0 \mathrm{a}$ & $9,5 \mathrm{a}$ \\
\hline $\mathrm{AQ}$ & $5,5 \mathrm{a}$ & - & - & $7,8 \mathrm{a}$ & $14,0 \mathrm{a}$ & $10,8 \mathrm{a}$ \\
\hline $\mathrm{I}+\mathrm{LO}$ & $2,8 \mathrm{~b}$ & $3,8 \mathrm{~b}$ & - & $8,5 \mathrm{a}$ & $12,0 \mathrm{a}$ & $6,2 a$ \\
\hline $\mathrm{I}+\mathrm{L} 1$ & $3,5 b$ & $3,5 b$ & - & $8,2 \mathrm{a}$ & $14,6 \mathrm{a}$ & $8,2 \mathrm{a}$ \\
\hline $\mathrm{I}+\mathrm{L} 2$ & $4,0 \mathrm{ab}$ & $4,5 b$ & - & $8,5 \mathrm{a}$ & $12,2 \mathrm{a}$ & $8,0 \mathrm{a}$ \\
\hline $\mathrm{I}+\mathrm{L} 3$ & $4,0 \mathrm{ab}$ & $7,8 \mathrm{a}$ & - & $7,8 \mathrm{a}$ & $19,6 \mathrm{a}$ & $10,8 \mathrm{a}$ \\
\hline $\mathrm{I}+\mathrm{AQ}$ & $3,5 b$ & $2,5 b$ & - & $9,0 \mathrm{a}$ & $17,5 \mathrm{a}$ & $7,0 \mathrm{a}$ \\
\hline
\end{tabular}

(1)Médias seguidas pela mesma letra, na coluna, dentro de cada época de avaliação, não diferem entre si pelo teste de Duncan a $5 \%$ de probabilidade. ${ }^{(2)} \mathrm{AQ}$ : adubação química completa $\left(60 \mathrm{~kg}\right.$ ha-1 de $\mathrm{P}_{2} \mathrm{O}_{5}, 60 \mathrm{~kg}$ ha-1 de $\mathrm{K}_{2} \mathrm{O}, 120 \mathrm{~kg}^{-1} \mathrm{e}^{-1}$ de $\mathrm{N}$ ); I+L0: soja com inoculação, sem aplicação de lodo de esgoto; I + L1: soja com inoculação, mais 1,5 t ha-1 de lodo de esgoto; I + L2: soja com inoculação, mais $3 \mathrm{t} \mathrm{ha}^{-1}$ de lodo de esgoto; I + L3: soja com inoculação mais $6 \mathrm{t} \mathrm{ha}^{-1}$ de lodo de esgoto; I + AQ: soja com inoculação, mais adubação química, exceto a nitrogenada. (3)Dados variáveis. judicado o processo da nodulação. No segundo ano agrícola, porém, as raízes de todas as plantas dos tratamentos que receberam inoculante apresentaram nódulos.

A maior produtividade em 2000/2001 foi obtida com as duas maiores doses de lodo (Tabela 6). Em média, esses tratamentos produziram cerca de 547 e $163 \mathrm{~kg} \mathrm{ha}^{-1}$ de grãos a mais, respectivamente, do que os tratamentos testemunha e com adubação mineral. O teor de $\mathrm{N}$ no solo parece não ter sido um fator limitante da produtividade, uma vez que no tratamento com inoculação que recebeu apenas $\mathrm{P}$ e K, a produtividade foi semelhante à do tratamento AQ.

Tabela 5. Nitrogênio total acumulado na parte aérea da soja cultivada em solo submetido aos tratamentos com adubos minerais e lodo de esgoto, em 2000/2001 (efeito direto) e 2001/ 2002 (efeito residual) $^{(1)}$.

\begin{tabular}{|c|c|c|c|c|}
\hline \multirow[t]{2}{*}{ Testemunha } & \multicolumn{2}{|c|}{ Ano $2000 / 2001$} & \multicolumn{2}{|c|}{ Ano $2001 / 2002$} \\
\hline & $32 \mathrm{DAE}$ & $64 \mathrm{DAE}$ & $32 \mathrm{DAE}$ & $71 \mathrm{DAE}$ \\
\hline & \multicolumn{4}{|c|}{$\mathrm{N}$ acumulado (mg planta $\left.{ }^{-1}\right)$} \\
\hline Testemunha & $37,2 \mathrm{c}$ & $143,3 \mathrm{c}$ & $162,8 b c$ & $173,3 d$ \\
\hline AQ & $56,7 \mathrm{bc}$ & $389,5 \mathrm{a}$ & $208,1 \mathrm{a}$ & $337,7 \mathrm{ab}$ \\
\hline $\mathrm{I}+\mathrm{L} 0$ & $49,8 b c$ & $200,3 \mathrm{c}$ & $140,5 \mathrm{c}$ & $198,9 \mathrm{~cd}$ \\
\hline $\mathrm{I}+\mathrm{L} 1$ & $67,6 \mathrm{~b}$ & $380,4 a$ & $153,5 b c$ & $268,5 b c$ \\
\hline $\mathrm{I}+\mathrm{L} 2$ & $67,7 b$ & $333,8 \mathrm{ab}$ & $160,0 \mathrm{bc}$ & $324,4 \mathrm{ab}$ \\
\hline $\mathrm{I}+\mathrm{L} 3$ & $96,3 \mathrm{a}$ & $343,6 \mathrm{ab}$ & $156,8 \mathrm{bc}$ & $356,8 \mathrm{a}$ \\
\hline $\mathrm{I}+\mathrm{AQ}$ & $57,6 \mathrm{~b}$ & $244,2 \mathrm{bc}$ & $172,0 \mathrm{~b}$ & $275,8 \mathrm{abc}$ \\
\hline
\end{tabular}

${ }^{(1)}$ Médias seguidas pela mesma letra, na coluna, dentro de cada época de avaliação, não diferem entre si pelo teste de Duncan a $5 \%$ de probabilidade. ${ }^{(2)} \mathrm{AQ}$ : adubação química completa (60 kg ha-1 de $\mathrm{P}_{2} \mathrm{O}_{5}$, $60 \mathrm{~kg}$ de $\mathrm{K}_{2} \mathrm{O}, 120 \mathrm{~kg} \mathrm{ha}^{-1}$ de $\mathrm{N}$ ); I + L0: soja com inoculação, sem aplicação de lodo de esgoto; I + L1: soja com inoculação, mais 1,5 t ha-1 de lodo de esgoto; I + L2: soja com inoculação, mais $3 \mathrm{t} \mathrm{ha}^{-1}$ de lodo de esgoto; I + L3: soja com inoculação, mais $6 \mathrm{t} \mathrm{ha}^{-1}$ de lodo de esgoto; I + AQ: soja com inoculação mais adubação química, exceto a nitrogenada.

Tabela 6. Efeito direto (2000/2001) e residual (2001/2002) de adubos minerais e lodo de esgoto na produtividade da soja ${ }^{(1)}$.

\begin{tabular}{|c|c|c|}
\hline Tratamentos $^{(2)}$ & Ano $2000 / 2001$ & Ano $2001 / 2002$ \\
\hline & \multicolumn{2}{|c|}{ - } \\
\hline Testemunha & $1.498 \mathrm{~d}$ & $2.685 b$ \\
\hline AQ & $1.877 \mathrm{ab}$ & $2.967 \mathrm{ab}$ \\
\hline $\mathrm{I}+\mathrm{L} 0$ & $1.554 \mathrm{~cd}$ & $2.629 \mathrm{~b}$ \\
\hline $\mathrm{I}+\mathrm{L} 1$ & $1.623 \mathrm{bcd}$ & $2.773 \mathrm{ab}$ \\
\hline $\mathrm{I}+\mathrm{L} 2$ & $2.079 \mathrm{a}$ & $3.099 \mathrm{a}$ \\
\hline $\mathrm{I}+\mathrm{L} 3$ & $2.002 \mathrm{a}$ & $3.118 \mathrm{a}$ \\
\hline $\mathrm{I}+\mathrm{AQ}$ & $1.814 \mathrm{abc}$ & $3.048 \mathrm{a}$ \\
\hline
\end{tabular}

(1)Médias seguidas pela mesma letra, na coluna, dentro de cada época de avaliação, não diferem entre si pelo teste de Duncan a $5 \%$ de probabilidade. (2)AQ: adubação química completa $\left(60 \mathrm{~kg} \mathrm{ha}^{-1}\right.$ de $\mathrm{P}_{2} \mathrm{O}_{5}, 60 \mathrm{~kg}$ ha-1 de $\mathrm{K}_{2} \mathrm{O}, 120 \mathrm{~kg} \mathrm{ha}^{-1}$ de N); I + L0: soja com inoculação, sem aplicação de lodo de esgoto; I + L1: soja com inoculação, mais $1,5 \mathrm{t} \mathrm{ha}^{-1}$ de lodo de esgoto; I + L2: soja com inoculação, mais $3 \mathrm{t} \mathrm{ha}^{-1}$ de lodo de esgoto; I + L3: soja com inoculação, mais 6 t ha-1 de lodo de esgoto; I + AQ: soja com inoculação, mais adubação química, exceto a nitrogenada. 
Nos tratamentos que não receberam adubação fosfatada (testemunha e I + L0), ou que a receberam em quantidades não suficientes (I + L1), a produtividade foi menor. No segundo ano de cultivo da soja, produtividade maior do que $3.000 \mathrm{~kg} \mathrm{ha}^{-1}$ foi obtida nos tratamentos I + AQ, I + L2 e I + L3 (Tabela 6). Esses dois últimos tratamentos superaram, respectivamente, em 141 e $423 \mathrm{~kg} \mathrm{ha}^{-1}$ de grãos, os tratamentos com adubação mineral e testemunha.

As produtividades da soja dos dois anos agrícolas demonstraram que tanto 3 quanto $6 \mathrm{t} \mathrm{ha}^{-1}$ de lodo foram suficientes para suprir as demandas da cultura em $\mathrm{P}$, quando comparados ao tratamento convencional (I + AQ). No segundo ano de cultivo, no tratamento $\mathrm{I}+\mathrm{L} 1$, aos 32 e $71 \mathrm{DAE}$, a despeito de os teores de P encontrados no solo não terem sido significativamente menores do que os do tratamento I + AQ, a produtividade da soja foi menor. Isto pode estar relacionado à deficiência de algum outro nutriente, ocasionada pela menor aplicação do composto orgânico.

Não houve diferença significativa entre os tratamentos, nos teores de $\mathrm{Fe}$ e Cu nos grãos da soja, nos dois cultivos. Os teores de Zn nos grãos não apresentaram diferenças significativas entre os tratamentos, no primeiro cultivo; no segundo cultivo, ele foi menor no tratamento que recebeu a menor dose de lodo (Tabela 7). Os teores de $\mathrm{Cd}, \mathrm{Pb}$ e Ni dos grãos não puderam ser determinados, porque suas concentrações estavam abaixo do limite de detecção, segundo o método usado por Carmo et al. (2000).

Tabela 7. Teores de alguns elementos químicos nos grãos de soja cultivada em solo submetido a tratamentos com adubos minerais e lodo de esgosto em 2000/2001 (efeito direto) e 2001/2002 (efeito residual)(1).

\begin{tabular}{|c|c|c|c|c|c|c|}
\hline \multirow[t]{2}{*}{ Tratamento $^{(2)}$} & \multicolumn{3}{|c|}{ Ano $2000 / 2001$} & \multicolumn{3}{|c|}{ Ano $2001 / 2002$} \\
\hline & $\mathrm{Fe}$ & $\mathrm{Zn}$ & $\mathrm{Cu}$ & $\mathrm{Fe}$ & $\mathrm{Zn}$ & $\mathrm{Cu}$ \\
\hline & & & $--(\mathrm{mg}$ & $-1)$ & & \\
\hline Testemunha & $150 \mathrm{a}$ & $41,1 \mathrm{a}$ & $11,1 \mathrm{a}$ & $87 a$ & $32,5 \mathrm{ab}$ & $9,4 a$ \\
\hline AQ & $160 \mathrm{a}$ & $37,2 \mathrm{a}$ & $-(3)$ & $134 \mathrm{a}$ & $32,3 \mathrm{ab}$ & $10,2 \mathrm{a}$ \\
\hline $\mathrm{I}+\mathrm{L} 1$ & $158 \mathrm{a}$ & $39,6 \mathrm{a}$ & $11,5 \mathrm{a}$ & $167 \mathrm{a}$ & $30,9 b$ & $9,0 \mathrm{a}$ \\
\hline $\mathrm{I}+\mathrm{L} 2$ & $141 \mathrm{a}$ & $39,8 \mathrm{a}$ & $10,6 \mathrm{a}$ & $128 \mathrm{a}$ & $34,1 \mathrm{a}$ & $9,6 \mathrm{a}$ \\
\hline $\mathrm{I}+\mathrm{L} 3$ & $147 \mathrm{a}$ & $40,0 \mathrm{a}$ & $11,0 \mathrm{a}$ & - & $34,6 \mathrm{a}$ & $9,0 \mathrm{a}$ \\
\hline
\end{tabular}

(1)Médias seguidas pela mesma letra, na coluna, dentro de cada época de avaliação, não diferem entre si pelo teste de Duncan a $5 \%$ de probabilidade. ${ }^{(2)} \mathrm{AQ}$ : adubação química completa (60 kg ha-1 de $\mathrm{P}_{2} \mathrm{O}_{5}, 60 \mathrm{~kg} \mathrm{ha}{ }^{-1}$ de $\mathrm{K}_{2} \mathrm{O}, 120 \mathrm{~kg} \mathrm{ha}^{-1}$ de $\mathrm{N}$ ); I + L1: soja com inoculação, mais $1,5 \mathrm{t} \mathrm{ha}^{-1}$ de lodo de esgoto; I + L2: soja com inoculação, mais $3 \mathrm{t} \mathrm{ha}^{-1}$ de lodo de esgoto; I + L3: soja com inoculação, mais $6 \mathrm{t} \mathrm{ha}^{-1}$ de lodo de esgoto. (3)Dados variáveis.

\section{Conclusões}

1. A necessidade de P da cultura da soja pode ser suprida pela adição de lodo de esgoto ao solo.

2. A aplicação de lodo de esgoto ao solo pode acarretar perdas de $\mathrm{NO}_{3}{ }^{-}$para o ambiente.

3. A adição de lodo de esgoto ao solo não ocasiona aumento nos teores de $\mathrm{Fe}, \mathrm{Zn}, \mathrm{Cu}, \mathrm{Cd}, \mathrm{Pb}$ e $\mathrm{Ni}$ nos grãos da soja.

\section{Agradecimentos}

À Fapesp (Fundação de Amparo à Pesquisa do Estado de São Paulo), pelo apoio financeiro.

\section{Referências}

ABD-ALLA, H.M.; YAN, F.; SCHUBERT, S. Effects of sewage sludge application on nodulation, nitrogen fixation, and plant growth of faba bean, soybean and lupin. Journal of Applied Botany, v.73, p.69-75, 1999.

ANGLE, J.S.; MADARIAGA, G.M.; HEGER, E.A. Sewage sludge effects on growth and nitrogen fixation of soybean. Agricultural Ecosystem and Environment, v.41, p.231-239, 1992.

BARBARICK, K.A.; IPPOLITO, J.A.; WESTFALL, D.G. Distribution and mineralization of biosolids nitrogen applied to dryland wheat. Journal of Environmental Quality, v.25, p.796801, 1996.

BERTON, R.S.; CAMARGO, O.A.; VALADARES, J.M.A.S. Absorção de nutrientes pelo milho em resposta à adição de lodo de esgoto a cinco solos paulistas. Revista Brasileira de Ciência do Solo, v.13, p.187-192, 1989.

BINDER, D.L.; DOBERMANN, A.; SANDER, D.H.; CASSMAN, K.G. Biosolids as nitrogen source for irrigated maize and rainfed sorghum. Soil Science Society of America Journal, v.66, p.531543, 2002.

CARMO, C.A.F. de S.; ARAUJO, W.S. de; BERNARDI, A.C. de C.; SALDANHA, M.F.C. Métodos de análise de tecidos vegetais utilizados na Embrapa Solos. Rio de Janeiro: Embrapa Solos, 2000. 41p. (Circular Técnica, 6).

DOU, H.; ALVA, A.K.; KHAKURAL, B.R. Nitrogen mineralization from citrus trees residues under different production conditions. Soil Science Society of America Journal, v.61, p.1226-1232, 1997.

EMBRAPA. Serviço Nacional de Levantamento e Conservação de Solos (Rio de Janeiro, RJ). Manual de métodos de análise de solos. 2.ed. Rio de Janeiro, 1997. 212p.

EMBRAPA. Serviço Nacional de Levantamento e Conservação de Solos (Rio de Janeiro, RJ). Sistema Brasileiro de Classificação de Solos. Rio de Janeiro, 1999. 412p.

GILLER, K.E.; WILSON, K.J. Environmental constraints to nitrogen fixation. In: GILLER, K.E.; WILSON, K.J. (Ed.). Nitrogen fixation in tropical cropping systems. Melksham, Wiltshire, UK: Redwood Press, 1991. p.199-214. 
HERNÁNDEZ, T.; MORAL, R.; PEREZ-ESPINOSA, A.; MORENO-CASELLES, J.; PEREZ-MURCIA, M.D.; GARCÍA, C. Nitrogen mineralisation potential in calcareous soils amended with sewage sludge. Bioresource Technology, v.83, p.213-219, 2002.

LINDSAY, B.J.; LOGAN, T.J. Field response of soil physical properties to sewage sludge. Journal of Environmental Quality, v.27, p.534-542, 1998.

MASCARENHAS, H.A.A.; TANAKA, R.T. Soja. In: RAIJ, B. van; CANTARELLA, H.; QUAGGIO, J.A.; FURLANI, A.M. Recomendações de adubação e calagem para o Estado de São Paulo. Campinas: Instituto Agronômico, 1996. p.202-203. (Boletim Técnico, 100).

MAZZARINO, M.J.; LAOS, F.; SATTI, P.; MOYANO, S. Agronomic and environmental aspects of utilization of organic residues in soils of the Andean-Patagonian region. Soil Science and Plant Nutrition, v.44, p.105-113, 1998.

NOVAIS, R.F.; SMYTH, T.J. Transformações de fósforo lábil em não-lábil. In: NOVAIS, R.F.; SMYTH, T.J. (Ed.). Fósforo em solo e planta em condições tropicais. Viçosa: Universidade Federal de Viçosa, 1999. 399p.

OLIVEIRA, F.C.; MARQUES, M.O.; BELLINGIERI, P.A.; PERECIN, D. Lodo de esgoto como fonte de macronutrientes para a cultura do sorgo granífero. Scientia Agricola, v.52, p.360367, 1995.

ROS, C.O. das; AITA, C.; CERETTA, C.A.; FRIES, M.R. Lodo de esgoto: efeito imediato no milheto e residual na associação aveia-ervilhaca. Revista Brasileira de Ciência do Solo, v.17, p.257261, 1993.

TEDESCO, M.J.; VOLKWEISS, S.J.; BOHNEN, H. Análise de solo, plantas e outros materiais. Porto Alegre: Universidade Federal do Rio Grande do Sul, 1985. (Boletim Técnico, 5).

VIEIRA, R.F. Sewage sludge effects on soybean growth and nitrogen fixation. Biology and Fertility of Soils, v.34, p.196-200, 2001.

VIEIRA, R.F.; CARDOSO, A.A. Variações nos teores de nitrogênio mineral em solo suplementado com lodo de esgoto. Pesquisa Agropecuária Brasileira, v.38, p.867-874, 2003.

VIEIRA, R.F.; TSAI, S.M.; TEIXEIRA, M.A. Utilização de lodo de esgoto na cultura do feijão: efeito sobre o crescimento e fixação simbiótica do $\mathrm{N}_{2}$. In: CONGRESSO NACIONAL DE PESQUISA DE FEIJÃO, 7, 2002, Viçosa. Anais. Viçosa: UFV, 2002. p.810-813.

WHITE, C.S.; LOFTIN, S.R.; AGUILAR, R. Application of biosolids to degraded semiarid rangeland: nine-year responses. Journal of Environmental Quality, v.26, p.1663-1671, 1997. 